\title{
Material Discrimination at High Spatial Resolution using Sub-300eV X-rays
}

\author{
P. Statham, J. Sagar, S. Burgess, J. Holland \\ Oxford Instruments NanoAnalysis, High Wycombe, Bucks, U.K.
}

Conventional X-ray analysis has relied on X-rays being generated by transitions that produce X-rays at a fixed energy characteristic of the atom involved and where the generated intensity from a bulk material is proportional to the mass fraction of the element. In the decades that followed Castaing's pioneering work on quantitative microprobe analysis with a wavelength dispersive spectrometer (WDS), many research groups worked on improving the algorithms for correcting for the effects of the matrix on excitation and absorption, the technology for the energy dispersive spectrometer (EDS) improved and there is now international consensus on how reliable element compositional analysis for atomic number $\mathrm{Z}>=11$ can be achieved with EDS at beam energies of $10-25 \mathrm{keV}$ [1]. For X-ray energies below $1 \mathrm{keV}$, quantitative analysis using $\mathrm{L}$ and $\mathrm{M}$ line emissions is less reliable because of anomalous absorption and chemical bonding effects [2] and at energies below $300 \mathrm{eV}$, the chemical environment of the atom has a significant influence even on K emissions. Such "chemical effects" were seen as a barrier to quantitative analysis and were the subject of considerable investigation in the 1960's (e.g. [3]). At the time, special purpose instruments were built for particular research projects and used electron beams that were not capable of high spatial resolution.

Quantitative analysis for sub-300eV X-rays still remains an elusive goal except for some restricted areas of application. However, in the last few years, some major technology advances have enabled new spectrometers to become available commercially that offer detection of X-rays to below $50 \mathrm{eV}$ in energy ("SXES" WDS [4] and "X-Max Extreme" EDS [5]) and can be mounted on electron columns that can provide high beam currents at low $\mathrm{kV}$ with a highly focussed beam. This has allowed investigators to discover new ways to characterise materials that are beyond the bounds of conventional microanalysis. SXES provides extremely good energy resolution $(0.2 \mathrm{eV})$ revealing the full emission profile and has enabled collection of new data that surpasses what was discovered in the 1960's. X-max Extreme resolution approaches $30 \mathrm{eV}$ fwhm at these energies but is much more sensitive so that good signals can be obtained at much smaller beam currents and considerably faster than SXES. Chemical state is impossible to determine from X-ray intensity alone but the shape of the X-ray emission profile can sometimes enable discrimination of materials in different chemical and crystallographic states. Figure 1 shows two spectra taken from graphite facets of different orientation that show shifts in $\mathrm{C} \mathrm{K} \alpha$ position consistent with the variation in shoulder due to $\pi$ bonding that has been shown by SXES [4]. However emission band changes in $\mathrm{Si} \mathrm{L}$ between pure $\mathrm{Si}$ and $\mathrm{TiSi}_{2}$ and in $\mathrm{B} \mathrm{K}$ between $\mathrm{CaB}_{6}$ and $\mathrm{LaB}_{6}$ that are detectable by SXES [6] are too subtle to be discriminated using X-max Extreme. Furthermore energy shifts between different chemical states can be very difficult to detect by EDS when there are large differences in peak to background ratio or absorption between different compounds.

Although accurate quantification with sub-300 eV X-rays may not be possible and chemical state analysis challenging, the high sensitivity of X-Max Extreme gives characteristic intensity signals that are still useful even with very low beam energies and low beam currents. Signals from Ca L are much stronger with windowless EDS and $\mathrm{Si} \mathrm{L}, \mathrm{Al} \mathrm{L}$ and $\mathrm{Mg} \mathrm{L}$ lines are excited even at $1 \mathrm{kV}$ so that material discrimination can be achieved for dimensions less than $10 \mathrm{~nm}$ [5]. The detection of several low energy $\mathrm{N}$ line emissions have now been demonstrated and Ce and La $\mathrm{N}$ emissions used for mapping 5-50 nm 
structures [7]. Li $\mathrm{K}$ is at the low energy limit and although obtaining a Li peak from pure metal is comparatively straightforward, some Li compounds dissociate under the beam [8]. Dissociation and migration of Li with formation of surface Li particles has also been seen in LiLaAlZr oxide electrolyte after exposure to a 500pA $5 \mathrm{kV}$ beam for 5 minutes [9]. However, with X-Max Extreme enough data can still be collected with a $100 \mathrm{pA} 2 \mathrm{kV}$ beam for 7 minutes and figure 2(b) shows there is very little evidence of any damage or surface particle formation and the Li distribution map in figure 2(c) is now a more faithful representation of the true distribution.

\section{References:}

[1] ISO standard (2011) ISO 22309:2011.

[2] JL Pouchou, Mikrochim. Acta [Suppl.] 13 (1996), p. 39.

[3] JE Holliday, "The Electron Microprobe", John Wiley \& Sons (1966), p. 3-22.

[4] H Takahashi et al, Microsc. Microanal. 20 (2014), p. 684.

[5] S Burgess et al, Microscopy Today, 2 (2017), p. 20.

[6] M Terauchi et al, JEOL News 47(1) (2012), p. 23.

[7] S Burgess et al, Microsc. Microanal. 22 (2016), p. 112.

[8] C MacRae et al, IOP Conf. Series: Materials Science and Engineering 304 (2017), p. 012010.

[9] J Sagar et al, Microsc. Microanal. 23 (2017), p. 1840.

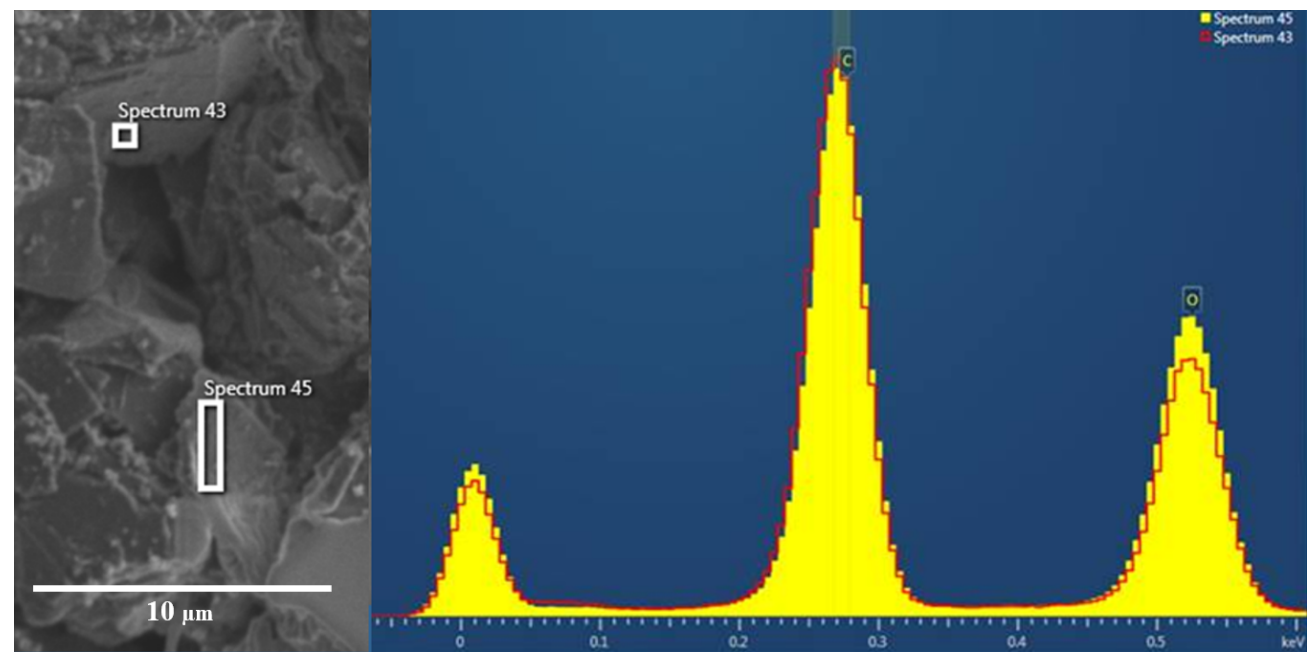

Figure 1. Spectra from Graphite facets at $3 \mathrm{kV}$. Detector is at bottom of field of view. C Ka for facet facing towards detector $(43$, red) is at lower energy than for facet facing away $(45$, yellow).

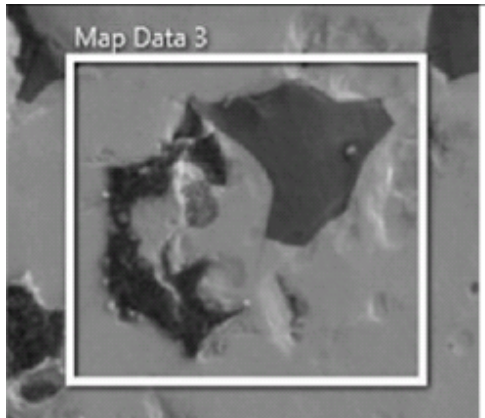

Figure 2. (a) Before map

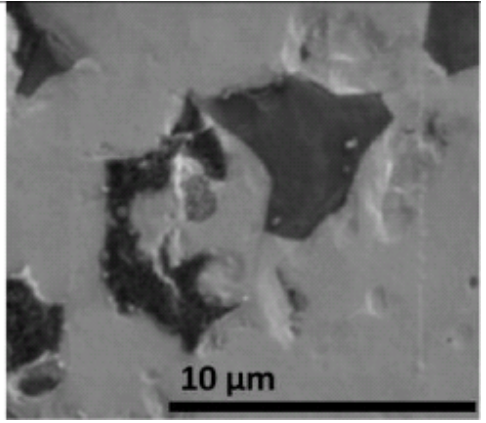

(b) After map

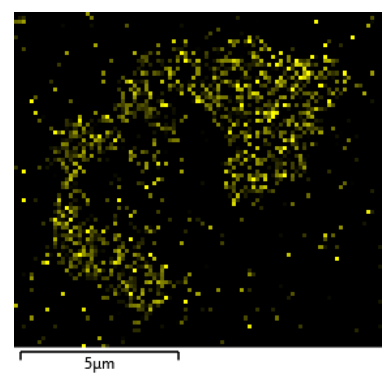

(c) Li map 100pA, 2kV, 7 minutes 